\title{
ON THE SOLUTIONS OF SECOND ORDER LINEAR DIFFERENTIAL EQUATIONS
}

\author{
CHOY-TAK TAAM
}

1. Let $P(x)$ and $Q(x)$ be complex-valued Lebesgue-measurable functions defined for all non-negative $x$, the functions $1 / P(x)$ and $Q(x)$ being of the class $L(0, R)$ for every positive $R$. A solution of the differential equation

$$
\left(P(x) W^{\prime}\right)^{\prime}+Q(x) W=0
$$

is an absolutely continuous function $W(x)$ such that $P(x) W^{\prime}(x)$ is equal almost everywhere to an absolutely continuous function $W_{1}(x)$, say, and that

$$
W_{1}^{\prime}(x)+Q(x) W=0
$$

is satisfied for almost all $x$. In the sequel only those solutions which are distinct from the trivial solution $(\equiv 0)$ shall be considered.

On the positive $x$-axis let $I$ be an interval which need not be closed or bounded. The equation (1.1) will be called disconjugate on $I$ if and only if no solution of (1.1) possesses more than one zero on $I$.

It is the purpose of this note to derive a general criterion (Theorem 1) for the differential equation (1.1) disconjugate on an interval and from which to prove a comparison theorem (Theorem 2). These results generalize those obtained previously by the author for the case $P(x)=1$ [2, Theorems 1 and 9]. When $P(x)=1$ and $Q(x)$ is real, an interesting discussion of disconjugate differential equations was given by $A$. Wintner [4].

The method of proof of Theorem 1 is a modification of that employed in [2, Theorem 1].

2. Write

$$
P(x)=p_{1}(x)+i p_{2}(x), \quad Q(x)=q_{1}(x)+i q_{2}(x),
$$

where $p_{1}, p_{2}, q_{1}$ and $q_{2}$ are real. We first prove the following general criterion.

THEOREM 1. Suppose that the following conditions are satisfied:

(1) $m=m(x)$ is a real-valued function absolutely continuous on every closed subinterval of $I$,

(2) for some real constants $j$ and $k, j p_{1}+k p_{2}$ is positive on $I$ and $1 /\left(j p_{1}+k p_{2}\right)$ belongs to the class $L$ on every closed subinterval of $I$,

Presented to the Society, April 25, 1953; received by the editors March 16, 1953. 
(3) $m(x)$ satisfies the inequality

$$
m^{\prime}+m^{2} /\left(j p_{1}+k p_{2}\right) \leqq-\left(j q_{1}+k q_{2}\right)
$$

almost everywhere on $I$.

Then (1.1) is disconjugate on $I$. Furthermore, if $I$ is closed at least at one end, there is a solution of (1.1) which does not vanish on $I$.

Proof. Suppose that the theorem is not true. Then there is a solution $W(x)$ which has at least two zeros $a$ and $b, a<b$, in $I$. We shall show that this leads to contradiction.

Let $W_{1}$ be the absolutely continuous function which is equal to $P W^{\prime}$ almost everywhere on $I$. Write

$$
W=u+i v, \quad W_{1}=u_{1}+i v_{1},
$$

where $u, v, u_{1}$, and $v_{1}$ are real. It is clear that

$$
u_{1}=p_{1} u^{\prime}-p_{2} v^{\prime}, \quad v_{1}=p_{2} u^{\prime}+p_{1} v^{\prime} .
$$

Separating the real and imaginary parts of (1.2), we get

$$
u_{1}^{\prime}=-q_{1} u+q_{2} v, \quad v_{1}^{\prime}=-q_{2} u-q_{1} v .
$$

The equalities in (2.4) and (2.5) hold almost everywhere on $I$. Let

$$
L=j\left(u u_{1}+v v_{1}\right)+k\left(u v_{1}-u_{1} v\right)-m\left(u^{2}+v^{2}\right) .
$$

Differentiating (2.6) and simplifying the result with (2.4) and (2.5), we have

$$
\begin{aligned}
L^{\prime}= & \left(j p_{1}+k p_{2}\right)\left(u^{\prime 2}+v^{\prime 2}\right)-2 m\left(u u^{\prime}+v v^{\prime}\right) \\
& -\left(m^{\prime}+j q_{1}+k q_{2}\right)\left(u^{2}+v^{2}\right)
\end{aligned}
$$

almost everywhere on $I$. Completing the squares, (2.7) yields

$$
\begin{aligned}
L^{\prime}= & \left(j p_{1}+k p_{2}\right)\left[\left(u^{\prime}-m u /\left(j p_{1}+k p_{2}\right)\right)^{2}\right. \\
& \left.+\left(v^{\prime}-m v /\left(j p_{1}+k p_{2}\right)\right)^{2}\right] \\
& -\left[m^{\prime}+m^{2} /\left(j p_{1}+k p_{2}\right)+j q_{1}+k q_{2}\right]\left(u^{2}+v^{2}\right) .
\end{aligned}
$$

The first term on the right-hand side of (2.8) is positive almost everywhere on $[a, b]$, otherwise $u$ and $v$ would be solutions of the differential equation

$$
y^{\prime}=m y /\left(j p_{1}+k p_{2}\right)
$$

on $[a, b]$, and, since $u$ and $v$ vanish at $a, u$ and $v$ must vanish identically on $[a, b]$, but this is impossible owing to the fact that $W \neq 0$. Integrating both sides of (2.8) from $a$ to $b$ and using (2.2), we have clearly 


$$
L(b)-L(a)>0 .
$$

Since $L$ vanishes at $a$ and $b$, we have contradiction. This proves that $W$ cannot possess two zeros on $I$ and hence (1.1) is disconjugate on $I$.

If $I$ is closed at the left end with end point $a$, then the argument above shows that $L(x) \geqq L(a)$ for all $x$ on $I$. Since $j p_{1}+k p_{2}$ is positive, $j$ and $k$ cannot both be zero. Suppose that $j$ is not zero. Let $W$ be a solution with

$$
W(a)=1, \quad W_{1}(a)=[m(a)+1] / j .
$$

For this solution it is easy to verify that $L(a)=1$. Hence $L(x) \geqq 1$ for all $x$ on $I$. Consequently, from (2.6), this solution does not vanish on $I$. The cases that $j=0, k \neq 0$, and $I$ is closed at the right end can be proved similarly. This completes the proof of Theorem 1 .

3. In this section, we shall prove a comparison theorem. Consider another differential equation

$$
\left(r(x) y^{\prime}\right)^{\prime}+f(x) y=0,
$$

where $r$ and $f$ are real-valued functions defined for all non-negative $x$, $r$ being positive, and $1 / r$ and $f$ belonging to $L(0, R)$ for every positive $R$. On the positive $x$-axis, let $I_{0}$ be an interval which is either closed or open, and if open need not be bounded.

THEOREM 2. Suppose that the following conditions are satisfied:

(1) (3.1) is disconjugate on $I_{0}$,

(2) for some real constants $j$ and $k$, the inequalities $j p_{1}+k p_{2} \geqq r$, $j q_{1}+k q_{2} \leqq f$ hold almost everywhere on $I_{0}$.

Then (1.1) is disconjugate on $I_{0}$. Furthermore, if $I_{0}$ is closed, there is a solution of (1.1) which does not vanish on $I_{0}$.

Proof. It is known that if (3.1) is disconjugate on $I_{0}$, there exists a real-valued function $m(x)$ which is absolutely continuous on every closed subinterval of $I_{0}$ and satisfying the inequality

$$
m^{\prime}+m^{2} / r \leqq-f
$$

almost everywhere on $I_{0}[3$, Theorem 1]. From (3.2) and condition (2) of the theorem, it is clear that

$$
m^{\prime}+m^{2} /\left(j p_{1}+k p_{2}\right) \leqq-\left(j q_{1}+k q_{2}\right)
$$

holds amost everywhere on $I_{0}$. The theorem then follows from Theorem 1.

4. In the following theorem, we consider the differential equation 


$$
\left(\left(j p_{1}+k p_{2}\right) y^{\prime}\right)^{\prime}+\left(j p_{1}+k p_{2}\right)^{-1} G^{2} y=0,
$$

where

$$
G(x)=2 \int_{a}^{x}\left(j q_{1}+k q_{2}+g\right) d x+A .
$$

THEOREM 3. Suppose that the following conditions are satisfied:

(1) $j, k$ and $A$ are real constants,

(2) $g=g(x)$ is real-valued, non-negative on $[a, b]$ and belongs to $L(a, b)$,

(3) $j p_{1}+k p_{2}$ is positive on $[a, b]$ and $\left(j p_{1}+k p_{2}\right)^{-1}$ belongs to $L(a, b)$,

(4) (4.1) is disconjugate on $[a, b]$.

Then (1.1) is disconjugate on $[a, b]$.

Proof. Since (4.1) is disconjugate on $[a, b]$, according to $[3$, Theorem 1], there exists a real-valued function $n(x)$ absolutely continuous on $[a, b]$ and satisfying

$$
n^{\prime}+n^{2} /\left(j p_{1}+k p_{2}\right) \leqq-G^{2} /\left(j p_{1}+k p_{2}\right)
$$

almost everywhere on $[a, b]$. Let $m=(n-G) / 2$. Using (4.2) and (4.3), it is easy to verify that $m$ satisfies (2.2) almost everywhere on $[a, b]$. The theorem then follows from Theorem 1.

Theorem 3 can be easily modified to apply to an open interval, bounded or unbounded.

Theorem 3 is a generalization of a theorem due to P. Hartman[1].

\section{REFERENCES}

1. P. Hartman, On linear second order differential equations with small coefficients, Amer. J. Math. vol. 73 (1951) pp. 955-962.

2. C. T. Taam, Non-oscillation and comparison theorems of linear differential equations with complex-valued coefficients, Portugaliae Mathematica vol. 12 (1953) pp. 5772.

3. - Non-oscillatory differential equations, Duke Math. J. vol. 19 (1952) pp. 493-497.

4. A. Wintner, On the non-existence of conjugate points, Amer. J. Math. vol. 73 (1951) pp. 368-380.

The Catholic University of America 